\title{
SLOW RADIATION-DRIVEN WIND SOLUTIONS OF A-TYPE SUPERGIANTS
}

\author{
M. CURÉ ${ }^{1}$, L. Cidale ${ }^{2,4}$, AND A. GRAnada ${ }^{2,3}$ \\ ${ }^{1}$ Departamento de Física y Astronomía, Facultad de Ciencias, Universidad de Valparaíso Av. Gran Bretaña 1111, Casilla 5030, Valparaíso, Chile; michel.cure@ uv.cl \\ ${ }^{2}$ Departamento de Espectroscopía, Facultad de Ciencias Astronómicas y Geofísicas, Universidad Nacional de La Plata (UNLP), and Instituto de Astrofísica La Plata, \\ CCT La Plata, CONICET-UNLP Paseo del Bosque S/N, 1900 La Plata, Argentina \\ ${ }^{3}$ Observatoire Astronomique de l'Université de Genève 51, Chemin des Maillettes, CH-1290, Sauverny, Switzerland \\ Received 2011 February 24; accepted 2011 May 20; published 2011 July 25
}

\begin{abstract}
The theory of radiation-driven winds succeeded in describing terminal velocities and mass-loss rates of massive stars. However, for A-type supergiants the standard m-CAK solution predicts values of mass loss and terminal velocity higher than the observed values. Based on the existence of a slow wind solution in fast rotating massive stars, we explore numerically the parameter space of radiation-driven flows to search for new wind solutions in slowly rotating stars that could explain the origin of these discrepancies. We solve the one-dimensional hydrodynamical equation of rotating radiation-driven winds at different stellar latitudes and explore the influence of ionization changes throughout the wind in the velocity profile. We have found that for particular sets of stellar and line-force parameters, a new slow solution exists over the entire star when the rotational speed is slow or even zero. In the case of slow rotating A-type supergiant stars, the presence of this novel slow solution at all latitudes leads to mass losses and wind terminal velocities which are in agreement with the observed values. The theoretical wind-momentum-luminosity relationship derived with these slow solutions shows very good agreement with the empirical relationship. In addition, the ratio between the terminal and escape velocities, which provides a simple way to predict stellar wind energy and momentum input into the interstellar medium, is also properly traced.
\end{abstract}

Key words: stars: mass-loss - stars: winds, outflows - supergiants

Online-only material: color figures

\section{INTRODUCTION}

The theory of radiation-driven winds or CAK theory (Castor et al. 1975) and its later improvements m-CAK (Friend \& Abbott 1986; Pauldrach et al. 1986) succeeded in describing the terminal velocities $\left(V_{\infty}\right)$ and mass-loss rates $(\dot{M})$ of very massive stars. Both CAK and m-CAK theories predict a tight relationship between the total mechanical momentum flow contained in the stellar wind outflow $\left(\dot{M} V_{\infty}\right)$ and the stellar luminosity $(L)$ of the mass-losing star, known as wind-momentum-luminosity (WM-L) relationship. The determination of a WM-L relationship for A and B supergiants (Asgs and Bsgs) is important because it would allow the use of these stars as extragalactic distance indicators (Bresolin \& Kudritzki 2004). This relationship had been first empirically found by Kudritzki et al. (1995) for a sample of galactic O-B-A supergiants and giants. Its existence was confirmed for most luminous O-type stars by Puls et al. (1996), who explained the difference of the WM-L relationship among Milky Way, Large Magellanic Cloud, and Small Magellanic Cloud in terms of their different abundances. Further observational studies of the WM-L relationship showed a strong dependence on the spectral type (Kudritzki et al. 1999) which was interpreted as an indication that the winds are driven by different sets of ions.

Although the CAK theory has proved to be successful in explaining the global mass-loss properties of $\mathrm{O}$ supergiants, the winds of Galactic mid-B supergiants are substantially weaker than predictions from the radiation-driven theory (Crowther et al. 2006). Studies involving UV data (Prinja et al. 2005) and radio observations (Benaglia et al. 2007) have found discrepancies between empirical and predicted mass-loss rates. In most of the cases the supersonic regime of the wind is modeled

\footnotetext{
${ }^{4}$ Member of the Carrera del Investigador Científico, CONICET, Argentina.
}

with a velocity structure parameterized with a classical $\beta$-type law, with the $\beta$ exponent in the range $1-3$, determined by fitting the $\mathrm{H} \alpha$ line profile.

Similarly to mid- and late-Bsgs, the $\mathrm{H} \alpha$ profile of Asg stars can be modeled with large $\beta$ values (Kudritzki et al. 1999). In addition, the winds of Asgs show values of $V_{\infty}$ of about a factor of three lower than the predicted values (Achmad et al. 1997). There is also observational evidence of a decrease of $V_{\infty}$ when increasing the effective escape velocity $V_{\text {esc }}$, where $V_{\text {esc }}=\sqrt{2 G M_{*}(1-\Gamma) / R_{*}}$ takes into account the effect of Thomson scattering on the gravitational potential through $\Gamma=\sigma_{\mathrm{e}} L_{*} /\left(4 \pi c G M_{*}\right)$, in clear contradiction with the standard radiation-driven wind theory (Verdugo et al. 1998). The existence of this negative slope was attributed to a change in the force multiplier parameter $\alpha$, either as a change in the ionization of the wind (via the parameter $\delta$ ) with distance or as a decoupling of the line-driven ions in the wind from the ambient gas (Achmad et al. 1997). The change in the ionization along the wind is often expressed by the difference between the parameters $\alpha$ and $\delta: \alpha_{\text {eff }}=\alpha-\delta$ (Kudritzki et al. 1999).

When Curé (2004) revisited the theory of steady rotating radiation-driven winds, he obtained an exact formula for the location of the critical (singular) points and for the mass-loss rate. He showed that there exists another family of singular points, in addition to the standard m-CAK solution family (hereafter fast wind solution, FWS) when the star's rotation $(V)$ is close to the critical rotation velocity $\left(V_{\text {crit }}\right)$, that is, $\Omega=V / V_{\text {crit }} \gtrsim 60 \%-70 \%$. The numerical solutions crossing through this other critical point family lead to winds with lower terminal velocities and higher densities ( $\sim 30$ times higher) than a non-rotating wind (FWS). He also found that for late B-type stars, these slow wind solutions (hereafter SWS) are also represented by a $\beta$-velocity law with $\beta>1$. 
Since the slow solutions might predict the formation of a circumstellar disk around fast rotating stars, Curé et al. (2005) modeled the density distribution of a rapidly rotating $\mathrm{B}[\mathrm{e}]$ supergiant (with $V_{\mathrm{rot}} \sim 200 \mathrm{~km} \mathrm{~s}^{-1} ; \Omega \gtrsim 0.6$ ) assuming a change in the line-force parameters due to the bi-stability jump. This model leads to a fast wind in the polar regions and slow outflows in the equatorial plane with density contrasts between the equator and the pole of about $10^{2}-10^{4}$ near the stellar surface $\left(r \lesssim 2 R_{*}\right)$ to values of $10^{1}-10^{2}$ up to a radii of $\sim 100 R_{*}$.

However, none of the previously found solutions (FWS and SWS) are able to explain the observed velocities and mass losses in Asgs that, often, present low rotation speeds $\left(V_{\mathrm{r} o t} \sim 40 \mathrm{~km} \mathrm{~s}^{-1} ; \Omega<0.4\right.$ ) and low outflow wind velocities. Nevertheless, we think that the large values of $\beta$ obtained empirically by Kudritzki et al. (1999) for Asgs and Bsgs could be related to the presence of another type of SWS.

Therefore, based on Achmad et al.'s (1997) hypothesis related to a change in the ionization of the wind, we investigate hydrodynamical solutions of rotating radiation winds for a wide range of line-force multiplier parameters.

In this work, we solve the one-dimensional (1D) hydrodynamical equation for rotating-driven winds and report the existence of a new kind of solutions obtained for slow rotating stars with high values of $\delta$ that resemble some of the properties of the SWS found by Curé (2004) for fast rotating stars.

In Section 2, we explore the influence of the ionization of the wind in the velocity profile of rotating radiation-driven flows, described by the line-force parameter $\delta$. We find that for a particular set of line-force multiplier parameters $k, \alpha$, and $\delta$ in the range of the effective temperature of Asgs, there exists a new wind solution that describes the properties of a weak outflow at all the stellar latitudes. This solution is obtained for low rotation rates, for instance $\Omega=V / V_{\text {crit }}=0.4$, and even for the case without rotation, $\Omega=0$. Discussion of our results and conclusions are given in Section 3.

\section{RESULTS}

We have solved numerically the 1D hydrodynamic equations of rotating radiation-driven winds and obtained the radial velocity wind solutions as a function of the latitude, as described by Curé (2004). We considered different rotational velocity rates and different stellar and line-force parameters. We adopt values for $\alpha=0.49-0.59$ and $k=0.37-0.86$ that are in the range of those computed by Abbott (1982), Vink et al. (1999), and Shimada et al. (1994), while the parameter $\delta$ was arbitrarily selected between 0.0 and 0.5 , in order to study the influence of changes in ionization throughout the wind. This selection range allows us to find the new hydrodynamical solutions.

Table 1 lists some of the models computed for a non-rotating $T_{\text {eff }}=10,000 \mathrm{~K}$ supergiant star with solar abundance. The first column indicates the model designation and shows whether the solution is the new slow solution (s) or the fast solution (f). Columns 2-5 quote a particular set of force multiplier parameters $(\alpha, k, \delta)$ and its corresponding $\alpha_{\mathrm{eff}}=\alpha-\delta$ value. For non-rotating A-type supergiants with low values of $\delta(\lesssim 0.25)$, we obtain the known m-CAK fast solutions at all latitudes, as expected. However, when $\delta \gtrsim 0.3$ the solution switches to a slow-acceleration mode. Regarding the velocity profile, this new slow solution is similar to the kind of slow solution reported by Curé (2004) for fast rotating stars, but the mass-loss rate of this new solution, and therefore the wind density profile, is much lower than both fast and slow (due to
Table 1

Wind Parameters for Non-rotating Stars $(\Omega=0)$ with $T_{\text {eff }}=10,000 \mathrm{~K}$, $\log g=2, R_{\star}=60 R_{\odot}, \log \left(L / L_{\odot}\right)=4.5$, and $V_{\mathrm{esc}}=319 \mathrm{~km} \mathrm{~s}^{-1}$

\begin{tabular}{lccccccc}
\hline \hline Model & $\alpha$ & $k$ & $\delta$ & $\alpha_{\text {eff }}$ & $\begin{array}{c}V_{\infty} \\
\left(\mathrm{km} \mathrm{s}^{-1}\right)\end{array}$ & $\begin{array}{c}\dot{M} \\
\left(M_{\odot} \mathrm{yr}^{-1}\right)\end{array}$ & $\begin{array}{c}D_{\text {mom }} \\
(\mathrm{cgs})\end{array}$ \\
\hline W01 (f) & 0.49 & 0.37 & 0.00 & 0.49 & 546 & $6.20 \times 10^{-8}$ & 27.22 \\
W02 (f) & 0.49 & 0.37 & 0.22 & 0.27 & 286 & $6.48 \times 10^{-9}$ & 25.96 \\
W03 (s) & 0.49 & 0.37 & 0.30 & 0.19 & 201 & $7.14 \times 10^{-10}$ & 24.85 \\
W04 (f) & 0.49 & 0.86 & 0.00 & 0.49 & 552 & $3.43 \times 10^{-7}$ & 27.97 \\
W05 (f) & 0.49 & 0.86 & 0.22 & 0.23 & 294 & $1.43 \times 10^{-7}$ & 27.31 \\
W06 (s) & 0.49 & 0.86 & 0.42 & 0.07 & 174 & $2.22 \times 10^{-10}$ & 24.28 \\
W07 (f) & 0.59 & 0.37 & 0.00 & 0.59 & 786 & $2.87 \times 10^{-7}$ & 28.04 \\
W08 (f) & 0.59 & 0.37 & 0.25 & 0.34 & 368 & $1.03 \times 10^{-7}$ & 27.27 \\
W09 (s) & 0.59 & 0.37 & 0.34 & 0.25 & 242 & $4.17 \times 10^{-8}$ & 26.69 \\
W10 (f) & 0.59 & 0.86 & 0.00 & 0.59 & 793 & $1.19 \times 10^{-6}$ & 28.66 \\
W11 (f) & 0.59 & 0.86 & 0.26 & 0.33 & 365 & $1.20 \times 10^{-6}$ & 28.33 \\
W12 (s) & 0.59 & 0.86 & 0.36 & 0.23 & 238 & $1.14 \times 10^{-6}$ & 28.12 \\
\end{tabular}

fast rotation) solutions. We were not able to find any new SWS in the interval $0.22<\delta<0.30$ for all latitudes.

Columns 6 and 7 list the corresponding terminal velocity and mass-loss rate. We can note that when increasing $\delta$ both the mass-loss rate and the terminal velocity decrease. The new solution yields $V_{\infty}<V_{\text {esc }}\left(=319 \mathrm{~km} \mathrm{~s}^{-1}\right)$, where $V_{\text {esc }}$ was computed assuming a value of $\sigma_{\mathrm{e}}=0.33 \mathrm{~cm}^{2} \mathrm{~g}^{-1}$ (Verdugo et al. 1998). Finally, the last column quotes the wind modified momentum parameter, $D_{\text {mom }}=\dot{M} V_{\infty} R_{\star}^{1 / 2}$ (Kudritzki et al. 1999).

The differences between the terminal velocities obtained with the fast and slow solutions are remarkable (see Table 1, Column 6 ). These new slow solutions yield values of $\dot{M}$ in the range from $10^{-6}$ to $10^{-10} M_{\odot} \mathrm{yr}^{-1}$ and $V_{\infty}$ between 150 and $250 \mathrm{~km} \mathrm{~s}^{-1}$. Thus, the ratio between fast and slow terminal velocities changes by a factor of 3.0.

In the case of a slowly rotating A-type supergiants, we solve the 1D hydrodynamical equations at all the latitudes and also found a family of SWS. In this paper, we study solutions that show the same topology for all stellar latitudes; however, we briefly want to mention a more general result related to the behavior of the solutions in rotating radiation-driven winds. When $\delta$ is larger than a certain minimum value (typically in the range $0.2-0.25$ ), the wind solution switches from the fast solution to this new slow-acceleration mode at equatorial regions remaining the fast solution at higher latitudes up to the pole. The zones dominated by the slow solution are enhanced when increasing $\delta$ from this minimum value, and when it reaches a particular value of $\delta>\delta_{\text {crit }}$ (e.g., $\sim 0.3$ ) this new slow solution prevails at all latitudes.

In a forthcoming paper, we will discuss in detail the behavior of the different hydrodynamical solutions with spectral types.

Table 2 lists some models for Asgs for which the fast and slow hydrodynamical solutions exist over the whole star. It quotes the $\delta_{\text {crit }}$ value that corresponds to the family of slow solutions at all latitudes. Columns 1-4 indicate the model designation and the stellar parameters: effective temperature, stellar surface gravity, and stellar radius, respectively. Columns 5-12 quote the force multiplier parameters and the values of the terminal velocities and mass fluxes in polar $\left(V_{\infty \text { pol }}, F_{m \text { pol }}\right)$ and equatorial $\left(V_{\infty \text { eq }}\right.$, $\left.F_{m \text { eq }}\right)$ directions. Columns 13-16 list the total mass-loss rate, $\log \left(L / L_{\odot}\right), V_{\text {esc }}$, and $D_{\text {mom }}$, respectively.

Figure 1 displays the behavior of the fast and slow solutions as a function of stellar latitude for a rotating star with $\Omega=0.4$. The models share the same stellar and wind parameters except 

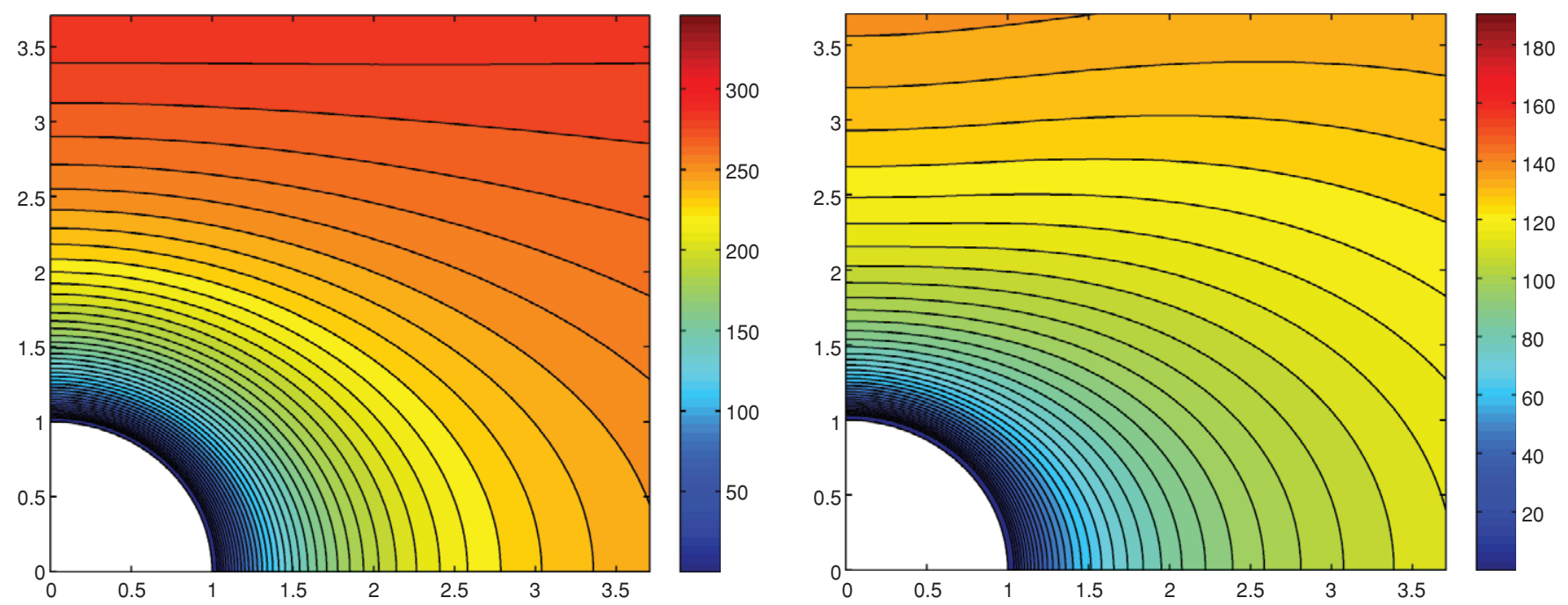

Figure 1. Latitude dependence of the wind velocity distribution for fast (left panel) and slow (right panel) solutions. The parameters of the solution correspond to model R08, except for the different values of $\delta$; fast solution is with $\delta=0.15$. The units of the axes are stellar radii and the abscissa defines the equatorial direction. The scale bars indicate the radial velocity in $\mathrm{km} \mathrm{s}^{-1}$.

(A color version of this figure is available in the online journal.)

Table 2

Slow Wind Solutions for Rotating Stars $(\Omega=0.4)$

\begin{tabular}{|c|c|c|c|c|c|c|c|c|c|c|c|c|c|c|c|}
\hline Model & $\begin{array}{l}T_{\text {eff }} \\
(\mathrm{kK})\end{array}$ & $\log g$ & $\begin{array}{c}R_{\star} \\
\left(R_{\odot}\right)\end{array}$ & $\alpha$ & $k$ & $\delta$ & $\alpha_{\mathrm{eff}}$ & $\begin{array}{c}V_{\infty \text { pol }} \\
\left(\mathrm{km} \mathrm{s}^{-1}\right)\end{array}$ & $\begin{array}{c}F_{\mathrm{mpol}} \\
\left(M_{\odot} \mathrm{yr}^{-1} \mathrm{sr}^{-1}\right)\end{array}$ & $\begin{array}{c}V_{\infty \mathrm{eq}} \\
\left(\mathrm{km} \mathrm{s}^{-1}\right)\end{array}$ & $\begin{array}{c}F_{\mathrm{meq}} \\
\left(M_{\odot} \mathrm{yr}^{-1} \mathrm{sr}^{-1}\right)\end{array}$ & $\begin{array}{c}\dot{M} \\
\left(M_{\odot} \mathrm{yr}^{-1}\right)\end{array}$ & $\log \left(L / L_{\odot}\right)$ & $\begin{array}{c}V_{\mathrm{esc}} \\
\left(\mathrm{km} \mathrm{s}^{-1}\right)\end{array}$ & $\begin{array}{l}D_{\text {mom }} \\
(\mathrm{cgs})\end{array}$ \\
\hline R01 (s) & 11 & 2 & 70 & 0.49 & 0.37 & 0.29 & 0.20 & 220 & $6.99 \times 10^{-10}$ & 192 & $9.09 \times 10^{-10}$ & $9.63 \times 10^{-9}$ & 4.80 & 286 & 26.03 \\
\hline R02 (s) & 11 & 2 & 70 & 0.49 & 0.86 & 0.33 & 0.16 & 209 & $2.98 \times 10^{-8}$ & 186 & $4.18 \times 10^{-8}$ & $4.21 \times 10^{-7}$ & 4.80 & 286 & 27.65 \\
\hline R03 (s) & 11 & 2 & 70 & 0.59 & 0.37 & 0.35 & 0.14 & 254 & $1.59 \times 10^{-8}$ & 226 & $1.99 \times 10^{-8}$ & $2.16 \times 10^{-7}$ & 4.80 & 286 & 27.44 \\
\hline R04 (s) & 11 & 2 & 70 & 0.59 & 0.86 & 0.36 & 0.13 & 255 & $5.36 \times 10^{-7}$ & 226 & $6.82 \times 10^{-7}$ & $7.33 \times 10^{-6}$ & 4.80 & 286 & 28.97 \\
\hline R05 (s) & 11 & 2 & 60 & 0.49 & 0.86 & 0.34 & 0.15 & 193 & $2.39 \times 10^{-8}$ & 173 & $3.45 \times 10^{-8}$ & $3.41 \times 10^{-7}$ & 4.67 & 317 & 27.49 \\
\hline R06 (s) & 11 & 2 & 60 & 0.59 & 0.37 & 0.35 & 0.24 & 237 & $1.34 \times 10^{-8}$ & 211 & $1.67 \times 10^{-8}$ & $1.82 \times 10^{-7}$ & 4.67 & 317 & 27.30 \\
\hline R07 (s) & 10 & 2 & 60 & 0.49 & 0.37 & 0.30 & 0.19 & 201 & $5.92 \times 10^{-11}$ & 178 & $7.78 \times 10^{-11}$ & $8.16 \times 10^{-10}$ & 4.50 & 308 & 24.88 \\
\hline R08 (s) & 10 & 2 & 60 & 0.49 & 0.86 & 0.33 & 0.16 & 194 & $2.18 \times 10^{-9}$ & 173 & $3.01 \times 10^{-9}$ & $3.09 \times 10^{-8}$ & 4.50 & 308 & 26.45 \\
\hline R09 (s) & 10 & 2 & 60 & 0.59 & 0.37 & 0.34 & 0.25 & 242 & $3.32 \times 10^{-9}$ & 214 & $4.11 \times 10^{-9}$ & $4.50 \times 10^{-8}$ & & 308 & 26.70 \\
\hline $\mathrm{R} 10$ (s) & 10 & 2 & 60 & 0.59 & 0.86 & 0.36 & 0.23 & 238 & $9.15 \times 10^{-8}$ & 212 & $1.15 \times 10^{-7}$ & $1.23 \times 10^{-6}$ & 4.50 & 308 & 28.14 \\
\hline R11 (s) & 10 & 1.7 & 80 & 0.49 & 0.37 & 0.30 & 0.19 & 167 & $1.40 \times 10^{-9}$ & 148 & $1.84 \times 10^{-9}$ & $1.93 \times 10^{-8}$ & 4.88 & 259 & 26.24 \\
\hline $\mathrm{R} 12$ (s) & 10 & 1.7 & 80 & 0.49 & 0.86 & 0.34 & 0.15 & 161 & $8.26 \times 10^{-8}$ & 144 & $1.21 \times 10^{-7}$ & $1.18 \times 10^{-6}$ & 4.88 & 259 & 28.01 \\
\hline $\mathrm{R} 13$ (s) & 10 & 1.7 & 80 & 0.59 & 0.37 & 0.34 & 0.25 & 198 & $3.01 \times 10^{-8}$ & 176 & $3.74 \times 10^{-8}$ & $4.07 \times 10^{-7}$ & 4.88 & 259 & 27.64 \\
\hline R14 (s) & 10 & 1.7 & 80 & 0.59 & 0.86 & 0.36 & 0.23 & 198 & $9.32 \times 10^{-7}$ & 176 & $1.20 \times 10^{-6}$ & $1.28 \times 10^{-5}$ & 4.88 & 259 & 29.14 \\
\hline $\mathrm{R} 15$ (s) & 9.5 & 2 & 60 & 0.49 & 0.37 & 0.30 & 0.19 & 202 & $2.26 \times 10^{-11}$ & 178 & $2.94 \times 10^{-11}$ & $3.10 \times 10^{-10}$ & 4.41 & 301 & 24.47 \\
\hline R16 (s) & 9.5 & 2 & 60 & 0.49 & 0.86 & 0.33 & 0.16 & 195 & $7.14 \times 10^{-10}$ & 174 & $9.95 \times 10^{-10}$ & $1.01 \times 10^{-8}$ & 4.41 & 301 & 25.96 \\
\hline $\mathrm{R} 17$ (s) & 9.5 & 2 & 60 & 0.59 & 0.37 & 0.34 & 0.25 & 242 & $1.51 \times 10^{-9}$ & 214 & $1.86 \times 10^{-9}$ & $2.04 \times 10^{-8}$ & 4.41 & 301 & 26.36 \\
\hline $\mathrm{R} 18$ (s) & 9.5 & 2 & 60 & 0.59 & 0.86 & 0.35 & 0.24 & 242 & $4.06 \times 10^{-8}$ & 213 & $5.10 \times 10^{-8}$ & $5.53 \times 10^{-7}$ & 4.41 & 301 & 27.79 \\
\hline R19 (s) & 9.5 & 1.7 & 100 & 0.49 & 0.37 & 0.30 & 0.19 & 184 & $5.91 \times 10^{-10}$ & 163 & $7.73 \times 10^{-10}$ & $8.14 \times 10^{-9}$ & 4.86 & 224 & 25.95 \\
\hline $\mathrm{R} 20$ (s) & 9.5 & 1.7 & 100 & 0.49 & 0.86 & 0.34 & 0.15 & 177 & $2.53 \times 10^{-8}$ & 158 & $3.60 \times 10^{-8}$ & $3.59 \times 10^{-7}$ & 4.86 & 224 & 27.58 \\
\hline $\mathrm{R} 21$ (s) & 9.5 & 1.7 & 100 & 0.59 & 0.37 & 0.34 & 0.25 & 219 & $1.70 \times$ & 194 & $2.11 \times 1$ & $2.30 \times 10^{-7}$ & 4.86 & 224 & 27.48 \\
\hline $\mathrm{R} 22(\mathrm{~s})$ & 9.5 & 1.7 & 100 & 0.59 & 0.86 & 0.36 & 0.23 & 216 & $4.90 \times 10^{-7}$ & 193 & $6.21 \times 10^{-7}$ & $6.68 \times 10^{-6}$ & 4.86 & 224 & 28.94 \\
\hline $\mathrm{R} 23$ (s) & 9 & 1.7 & 100 & 0.49 & 0.37 & 0.33 & 0.16 & 175 & $1.49 \times 10^{-10}$ & 157 & $2.11 \times 10^{-10}$ & $2.10 \times 10^{-9}$ & 4.76 & 201 & 25.35 \\
\hline $\mathrm{R} 24$ (s) & 9 & 1.7 & 100 & 0.49 & 0.86 & 0.33 & 0.16 & 179 & $2.77 \times 10^{-8}$ & 159 & $3.89 \times 10^{-8}$ & $3.91 \times 10^{-7}$ & 4.76 & 201 & 27.63 \\
\hline $\mathrm{R} 25$ (s) & 9 & 1.7 & 100 & 0.59 & 0.37 & 0.34 & 0.25 & 219 & $1.70 \times 10^{-8}$ & 194 & $2.11 \times 10^{-8}$ & $2.30 \times 10^{-7}$ & 4.76 & 201 & 27.48 \\
\hline R26 (s) & 9 & 1.7 & 100 & 0.59 & 0.86 & 0.36 & 0.23 & 217 & $4.90 \times 10^{-7}$ & 193 & $6.21 \times 10^{-7}$ & $6.67 \times 10^{-6}$ & 4.76 & 201 & 28.94 \\
\hline
\end{tabular}

for $\delta$ : the fast solution was computed with $\delta=0.15$ and the slow solution with $\delta=0.30$ (we chose as example model R08 from Table 2).

Finally, we want to remark that the theoretical wind parameters $\left(\dot{M}, V_{\infty}\right)$ related to this new SWS are in agreement with those observed in Asg stars (Achmad et al. 1997; Verdugo et al. 1998, 1999; Kudritzki et al. 1999). Moreover, the computed values for $D_{\text {mom }}$ using these new slow solutions follow the trend of the observed WM-L relationship for Asgs (as it is shown in Figure 2). A good agreement was obtained with the values re- ported by Abbott (1982), $\alpha=0.59, k=0.37$; however, models with a different set of line-force parameters, i.e., $\alpha=0.49$, $k=0.86$, fit, as well.

We also find that this new theoretical SWS predicts a decreasing relation of $V_{\infty} / V_{\text {esc }}$ with respect to $V_{\text {esc }}$ with the same observational trend found by Verdugo et al. (1998). Figure 3 shows our numerical results together with observational data obtained from the literature. The triangles pointing downwards and crosses (red symbols) represent the observational data of Verdugo et al. (1999); the crosses indicate terminal velocities 


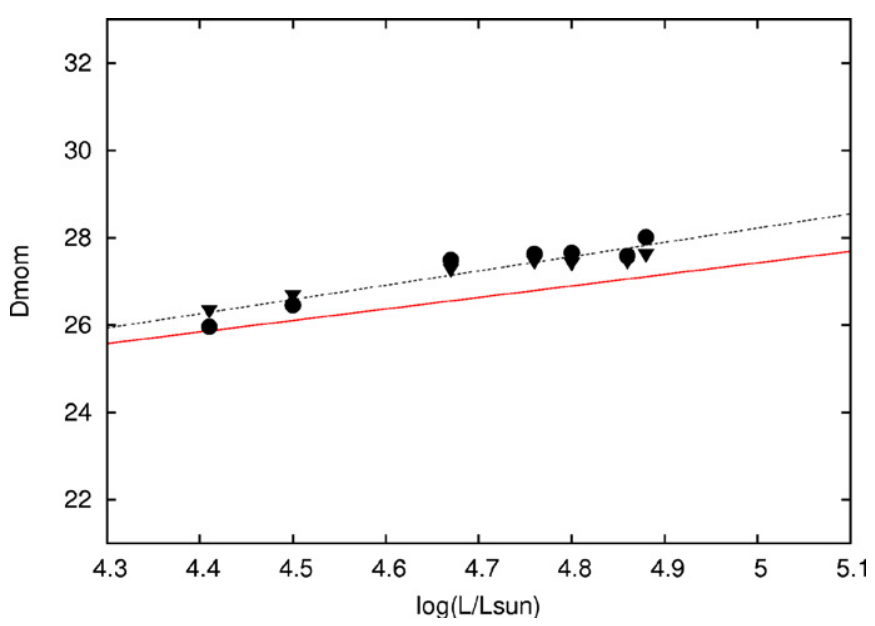

Figure 2. WM-L relationship derived from theoretical data computed from new slow wind models with $\Omega=0.4$ and the following sets of parameters, $\alpha=0.59$; $k=0.37$ (black triangles pointing downwards) and $\alpha=0.49 ; k=0.86$ (black circles), see Table 2. The theoretical WM-L relationship (dashed line) shows a good agreement with the observational relationship (red solid line) taken from Kudritzki et al. (1999).

(A color version of this figure is available in the online journal.)

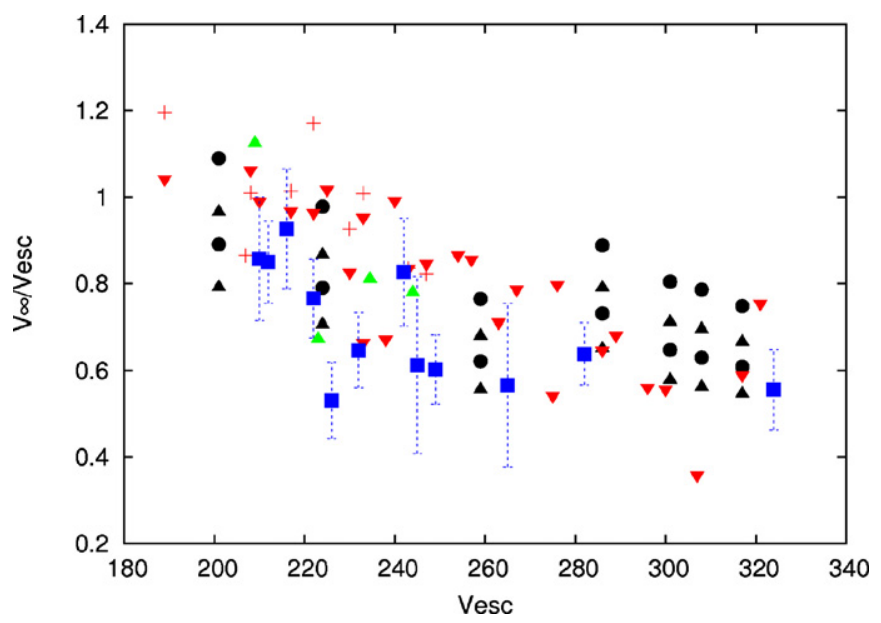

Figure 3. Relation between $V_{\infty} / V_{\text {esc }}$ versus $V_{\text {esc }}$ corresponding to polar (black circles) and equatorial (black triangles) slow solutions. Triangles pointing downwards and crosses (red symbols) represent the observational data taken from Verdugo et al. (1998); the crosses indicate terminal velocities obtained from saturated P Cygni UV lines whereas the triangles pointing downwards correspond to values determined by means of discrete absorption components; triangles pointing upwards (green) correspond to terminal velocities from Kudritzki et al. (1999); squares (blue) represent the measurements provided by Achmad et al. (1997) with their error estimates. Slow wind solution follows the same trend of the observations.

(A color version of this figure is available in the online journal.)

obtained from saturated P Cygni UV lines whereas the triangles represent the values obtained by means of discrete absorption components; the green triangles pointing upwards correspond to terminal velocities from Kudritzki et al. (1999); the blue squares represent the measurements provided by Achmad et al. (1997) with their error estimates. Our slow solution results are plotted in black symbols (circles/triangles for polar/equatorial directions) and nicely follow the same trend.

\section{DISCUSSION AND CONCLUSIONS}

Previous studies on radiation-driven winds based on 1D highrotating early-type stars $(\Omega>60 \%-70 \%)$ carried out by Curé (2004) demonstrated the existence of SWS. These types of solutions predict higher mass-loss rates and lower flow speeds at the equatorial plane than those of the polar zones and, as a consequence, a disk-like structure can be formed. In the present work, we explored numerically the parameter space of radiationdriven flows, particularly for high values of $\delta$, and found a new kind of wind solution for slowly rotating stars $(\Omega<40 \%$ and even without rotation). In order to distinguish the difference between the new solutions reported here and those obtained by Curé (2004), we propose to call them "low- $\Omega$, high- $\delta S W S$ ” and "high- $\Omega$, low- $\delta S W S$ ", respectively.

Although the low- $\Omega$, high- $\delta$ SWS were computed using $1 \mathrm{D}$ hydrodynamic equations, they provide a complete understanding of the dynamical outflow in slow rotating stars and settled a solid basis for the computation of multidimensional hydrodynamic models. In our particular case, the 1D approximation is a good approach to describe the slow wind properties of Asgs since the deformation of the star due to a slow rotational speed can be neglected. On the other hand, in a slow rotating star the density contrast between the equator and the pole is very low and, therefore, the wind would present a quasi-spherical distribution.

We found that low- $\Omega$, high- $\delta S W S$ properly trace the ratio between the terminal and escape velocities, which provides a simple way to predict stellar wind energy and momentum input into the interstellar medium. In addition, the new solutions follow the observational trend of $V_{\infty} / V_{\text {esc }}$ versus $V_{\text {esc }}$ reported by Verdugo et al. (1998). Our results support the Verdugo et al. (1998) hypothesis stating that the negative slope of the latter relation could be linked to the degree of ionization and the density of the wind.

Moreover, the theoretical WM-L relationship derived with low- $\Omega$, high- $\delta$ SWS shows a good agreement with the empirical relationship and brings back the idea of using these stars as extragalactic distance indicators (Bresolin \& Kudritzki 2004).

Taking into account the previous results, we think that low- $\Omega$, high- $\delta S W S$ might help to understand the long-standing problem of weak winds (see, e.g., Puls et al. 2008) because these solutions predict, besides slower terminal velocities, values of mass-loss rates that might be some hundred times lower than the standard or fast solutions, which precisely corresponds to the observed discrepancy between theory and observations.

The advantage of the model is that the new results stand on a radiation-driven wind characterized by the line-force parameters. Although the solution was obtained using ad hoc values of the parameter $\delta$, the calculation of this parameter should be revisited in order to introduce the effects of the variation of the ionization of the wind with distance. Future experiments to search for high values of the parameter $\delta$ in Asgs could be performed fitting line spectral features of different degree of ionization with synthetic line profiles computed with the radiative transfer equation and the new hydrodynamical wind model.

We thank the referee for helpful comments on our manuscript. M.C. acknowledges financial support from Centro de Astrofísica de Valparaíso. L.C. acknowledges financial support from the Agencia de Promoción Científica y Tecnológica (BID 1728 OC/ AR PICT 111), from CONICET (PIP 0300), and the Programa de Incentivos G11/089 of the Universidad Nacional de La Plata, Argentina.

\section{REFERENCES}

Abbott, D. C. 1982, ApJ, 259, 282

Achmad, L., Lamers, H. J. G. L. M., \& Pasquini, L. 1997, A\&A, 320, 196 
Benaglia, P., Vink, J. S., Martí, J., Apellániz, J. M., Koribalski, B., \& Crowther, P. A. 2007, A\&A, 467, 1265

Bresolin, F., \& Kudritzki, R. P. 2004, in Origin and Evolution of the Elements, ed. A. McWilliam \& M. Rauch (Cambridge: Cambridge Univ. Press), 283

Castor, J. I., Abbott, D. C., \& Klein, R. I. 1975, ApJ, 195, 157

Crowther, P. A., Lennon, D. J., \& Walborn, N. R. 2006, A\&A, 446, 279

Curé, M. 2004, ApJ, 614, 929

Curé, M., Rial, D. F., \& Cidale, L. 2005, A\&A, 437, 929

Friend, D. B., \& Abbott, D. C. 1986, ApJ, 311, 701

Kudritzki, R., Lennon, D. J., \& Puls, J. 1995, in Science with the VLT, ed. J. R. Walsh \& I. J. Danziger (Berlin: Springer), 246

Kudritzki, R. P., Puls, J., Lennon, D. J., Venn, K. A., Reetz, J., Najarro, F., McCarthy, J. K., \& Herrero, A. 1999, A\&A, 350, 970
Pauldrach, A., Puls, J., \& Kudritzki, R. P. 1986, A\&A, 164, 86

Prinja, R. K., Massa, D., \& Searle, S. C. 2005, A\&A, 430, 41

Puls, J., Vink, J. S., \& Najarro, F. 2008, A\&AR, 16, 209

Puls, J., et al. 1996, A\&A, 305, 171

Shimada, M. R., Ito, M., Hirata, B., \& Horaguchi, T. 1994, in IAU Symp. 162, Pulsation; Rotation; and Mass Loss in Early-Type Stars, ed. L. A. Balona, H. F. Henrichs, \& J. M. Le Contel (Dordrecht: Kluwer), 487

Verdugo, E., Talavera, A., \& Gómez de Castro, A. I. 1998, Ap\&SS, 263, 263

Verdugo, E., Talavera, A., \& Gómez de Castro, A. I. 1999, A\&AS, 137, 351

Vink, J. S., de Koter, A., \& Lamers, H. J. G. L. M. 1999, A\&A, 350, 181 